تأثير تطبيق نظام التتبع على صادرات مصر من العنب

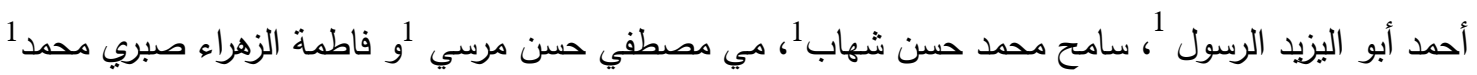

إمكانيات وسائل زيادة النصيب السوقي بها. وفتح أسواق جديدة

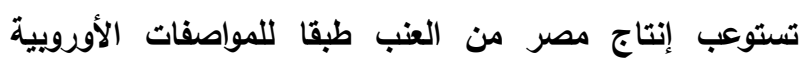
والمعايير الأوروبية لضمان استمرار الصادرات المصرية الزراعية

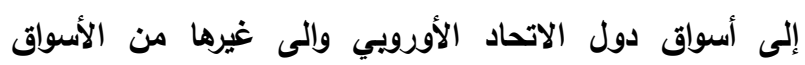

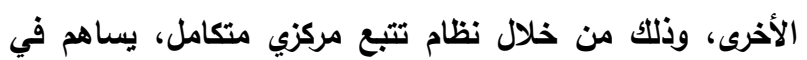

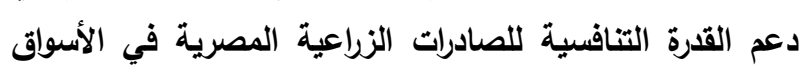

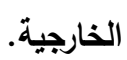

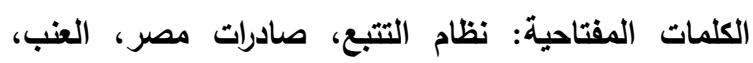
الاتحاد الأوروبي، مؤثرات التنافسية.

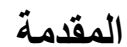

تعتبر سلامة الأغذية مصدر قلق عالمي بسبب عدد من

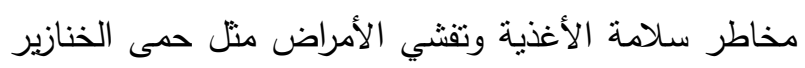

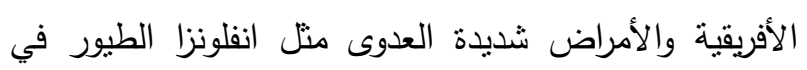
الدواجن ومرض الفم في الماشية ووجود الديوكسين والكائنات

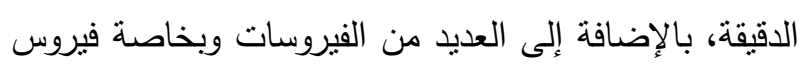

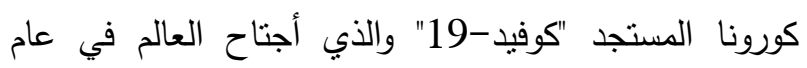

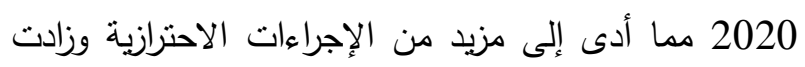
الحاجة إلى ضمان الجودة والسلامة فيما يتعلق بالأغذية الإنية

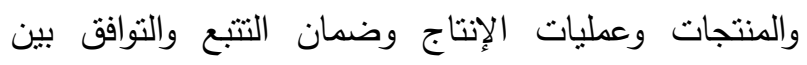
تدابير سلادة الأغذية. وقد حرصت القات وطيادة السياسية في

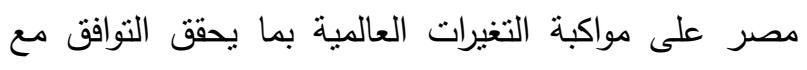
المعايير العالمية لسلامة الغذاء وذلك من خلال إنثاء هيئة متخصصة معنية بسلامة الغذاء (قانون الهيئة القومية لسلامة الفاء الغذاء، 2017) وتضع الهيئة نظاما لتتبع الأغذية يوضح الغئح التزامات كافة الأطراف في جميع مراحل الإنتاج والتجهيز والتصنيع والتعبئة والتداول حتى وصول المنتج إلى المستهلات،

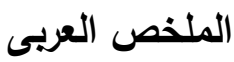

استهدف البحث التعرف على تأثير تطبيق نظام التتبع في زيادة تنافسية ونمو الصادرات المصرية من العب لاول الاتحاد

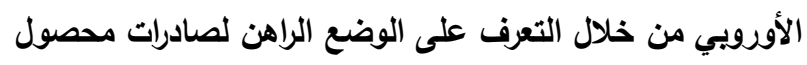

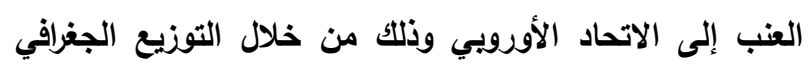

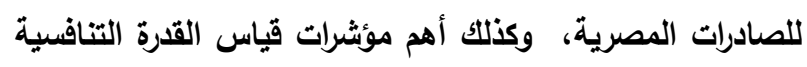

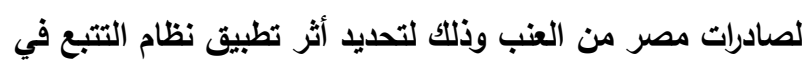

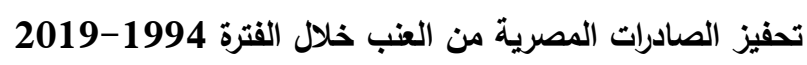

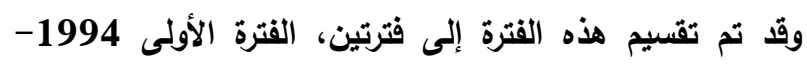
2006 وهي فترة ما قبل تطبيق نظام التتبع، والفترة الثانية 2007 - 2019 وهي فترة ما بعد تطبيق نظام التتبع للحاصلات الزراعية التصديرية في مصر.

وقد توصل البحث إلى عدد من النتائج، من أهمها ما يلي:

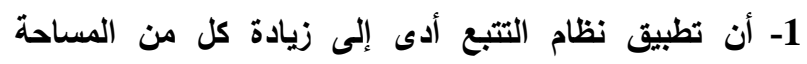
والإنتاج والإنتاجية لمحصول العنب المصري.

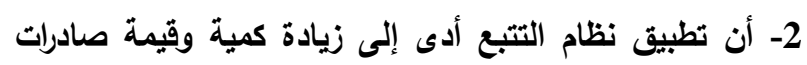
العنب لاول العالم وكنلك كمية وقيمة صادرات العبب لدول الاتحاد الأورويب. 3- الأسواق التصديرية للعب المصري تتركز بشكل كبير بين عدد

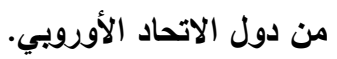

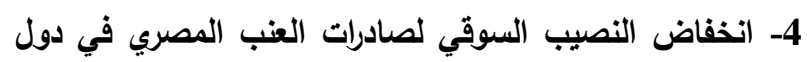

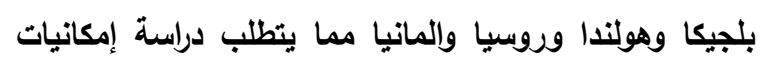
زيادة النصيب السوقي بتلك الدول. وأوصى البحث بضرورة المحافظة على الأسواق الاستيرادية

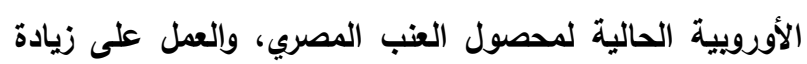

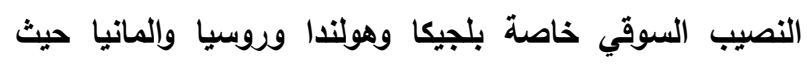
النصيب السوقي للعنب المصري فيها منذفض مما يتطب دراسة

معرف الوثيقة الرقمى:10.21608/asejaiqjsae.2021.187000 1اقسم الاقتصاد وادارة الأعمال الزراعية- كلية الزراعة- جامعة الإسكندرية

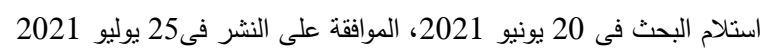


العنب المصري إلى الاتحاد الأوروبي، والتوزيع الجغرافي للصادرات المصرية من العنب وكنلك أهم مؤشرات قياس الاس الترني

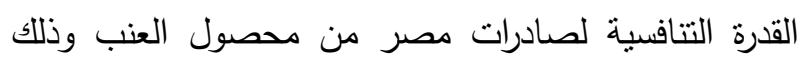
لتحديد أثنر تطبيق نظام التتبع في تحفيز الصادرات المصرية من العنب خلال الفترة 1994-2019 وقد نم تقسيم هذه الفترة إلى فترتين، الفترة الأولى خلف الفرة 1994-2006 وهي فترة ما قبل تطبيق نظام التتبع، والفترة الثانية 2007فترة ما بعد نطبيق نظام التتبع للحاصلات الزراعية التصديرية في مصر. أسلوب البحث ومصادر البيانات: تم استخدام الأساليب التحليلية الإحصائية الوصفية والتي

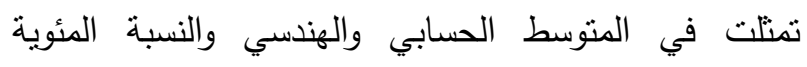

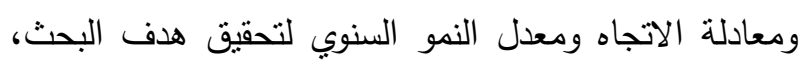

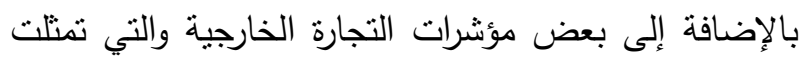

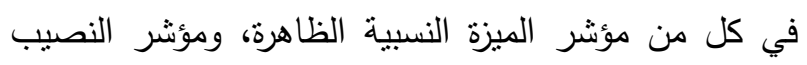
السوقي، كما نم استخدام أساليب العرض الجدولي ولئية والبياني. وفيما يلي عرض للمؤشرات المستخدمة في البحث: مؤثرات قياس القدرة التنافسية:

1. مؤشر الميزة النسبية الظاهرة: Revealed Comparative Advantage Index يقيس مؤشر الميزة النسبية الظاهرة (RCA) النصيب

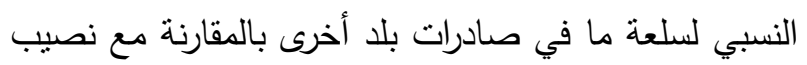

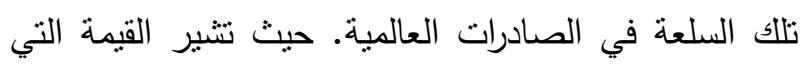
تقل فيها قيمة المؤشر عن 1 إلى عدم وجود ميزة نسبية

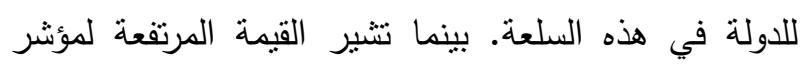

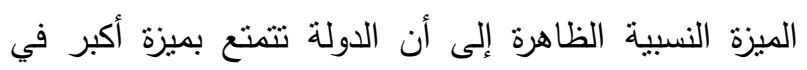

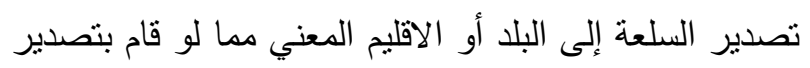
سلعة ذات قيمة أدنى للمؤشر المذكور.

$$
R C A_{j}=\frac{X_{e}^{j}}{X_{e}^{a}} \div \frac{X_{w}^{j}}{X_{w}^{a}}
$$

وهذا بدوره يؤدي إلى تطبيق نظاما لتتبع الصادرات الزراعية

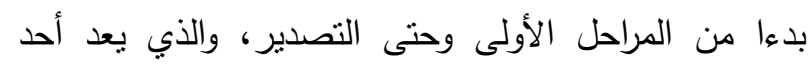
أدوات نجاح السياسة التصديرية لهصر حيث وليث يعمل تطبيق

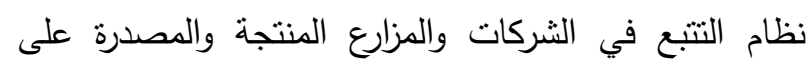
تحسين جودة الصادرات الزراعية المصرية (البطران، 2019)، ويعد العنب أهم محاصيل الفاكهة التصديرية في الترابة

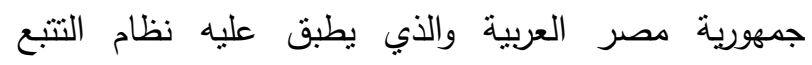
وبخاصة دول الاتحاد الأوروبي، كما يحتل العنب الترتيب لئل

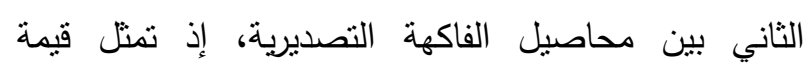
صادراته نحو 3.75\% من إجمالي قيمة الصادرات الزراعية كمنوسط للفترة 1994- 2019. إلا أن صادرات العنب المصري تمثل نسبة ضئيلة من الإنتاج المحلي.

\section{المشكلة البحثية}

على الرغم من أهمية محصول العنب بالنسبة لمحاصيل الفاكهة التصديرية، حيث يأتي في الترتيب الثاني بعد البرتقال

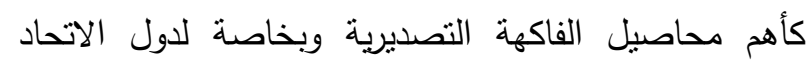

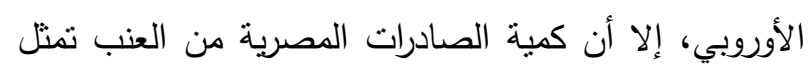
نسبة ضئيلة من الإنتاج الدحلي، وقد وضع الاتحاد الإداد الأوروبي

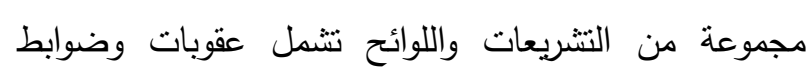

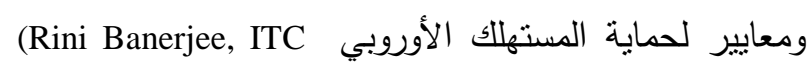

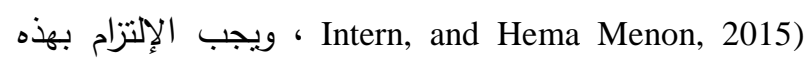

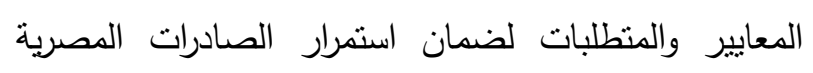
الزراعية إلى هذه الأسواق والى غيرها من الأسواق الأخرى،

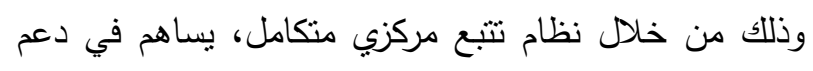

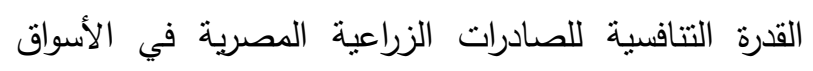

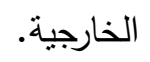

\section{الاهداف البحثية}

بستهدف البحث بصفة أساسية التعرف على تأثير تطبيق

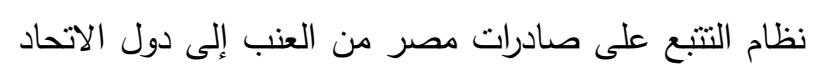
الأوروبي، وذلك من خلام دراسة الوضع الراهن لمحصول 
2004 تم أنشاء مشروع تثبع الصادرات الزراعية ETRACE (Egyptian Traceability Center for AgroIndustrial Export) التنافسية وزيادة الصادرات وتحسين جودة المنتج وسلامته حيث يقوم المشروع بمساعدة منتجي ومصدري المحاصيل الزراعية من جميع القطاعات العام والخاص والجمعيات الأهلية لضمان جودة المحاصيل الزراعية وسلامتها مع هع تطبيق نظام التتبع بحيث يتم التوافق مع اللوائح الخاصة

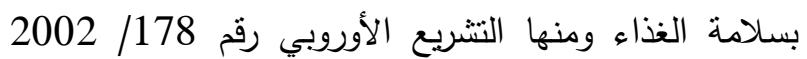
(المنظمة العربية للتتمية الزراعية، 2007). أهمية التتبع: يمكن إجمال فوائد نظام التتبع في الآتي(عبد العال، :(2017 1- إمكانية تتبع واسترجاع المنتج الزراعي والزراعي المصنع. 2- ضمان سلامة المنتج وعدم خلط منتج سليم بأخر نالف. 3- التوافق مع القوانين المحلية والدولية. 4- إرضاء العملاء وزيادة المبيعات.

\section{التتائسج والمناقشة}

1-الوضع الإنتاجي للعنب في مصر: تطور مساحة العنب المصري:

بدراسة تطور مساحة محصول العنب في جمهورية مصر

العربية خلال الفترة 1994-2019، أتضـح من الجدول (1) أنها نتراوح بين حد أدنى بلغ حوالي 121.54 ألف فدان عام 1995، وحد أقصى بلغ حوالي 193.28 ألف فدان عام 2017 وبمتوسط سنوي بلغ حوالي 155.21 ألف فدان. وتتير بيانات الجدول (2) أن مساحة محصول العنب اتجهت للزيادة خلال الفترة الأولى بمقار زيادة سنوي معنوي بلغ حوالي 2.67 ألف فدان، وبمعامل اختلاف بلغ نحو 8.94 وهو ما يشير إلى عدم وجود نشتت في مساحة محصول العنب خلال تللك الفترة، وبمعدل نمو سنوي معنوي إحصائيا عند مستوى 0.05 قدر بنحو 2\%، كما أن مساحة
RCA بالمقارنة بالدول المنافسة. قيمة صادرات الدولة من السلعة إلى العالم الخارجي. X قيمة الصادرات الزراعية للدولة إلى العالم الخارجي. X إجمالي قيمة صادرات العالم من السلعة الزراعية. إجمالي قيمة الصادرات الزراعية العالمية. 2. مؤثر النصيب السوقي:Market Share Index يبين النصيب السوقي مدى قدرة الصادرات على تغطية منطلبات الأسواق الخارجية وزيادة الإمكانات التصديرية لها، وهذا يعد من الآيات المهمة في رسم السياسات الإنتاجية والتصديرية والذي بنعكس بدوره على تحقيق الأهداف الإستراتيجية الزراعية.

وقد اعتمد البحث على البيانات الثانوية المنشورة من الثبكة الدولية للأمم المتحدة Comtrade ومنظمة الأغذية والزراعة للأمم المتحدة FAO وذلك خلا الفترة 1994.2019

\section{نظام التتبع: نشأته وتعريفه}

اهتمت المنظمة الدولية لتوحيد المقاييس بموضوع التتبع

قبل حدوث فضيحة البقر المجنون، إذ عرفته وفق معيار آيزو 8402 الذي صدر عام 1986 بأنه " القدرة على تتبع تاريخ أو استخدام أو مكان الثيء موضوع البحث الذي قد يتمثل في سلعة، أو نشاط، من خلال تحقق مسجل"، وفي النسخة الأخيرة للمعيار نفسه التي صدرت عام 1994، أدخلت بعض كل ولف التعديلات على التعريف بأنه "القدرة على تتبع ناريخ أو

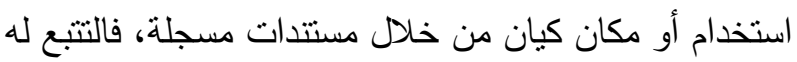
القدرة على إيجاد معلومات بشأن كيان معين قد يتمثل في مادة أولية أو آلة، أو مرحلة من مراحل التصنيع، أو منتج، مما يجعله أداة للوصول إلى المعلومات الخاصة بمراحل مره التحويل، وآلات القياس، والمنتجات نفسها من خلال وثائق ورقية والكترونية ورقمية مسجلة (بلعباس، 2018)، وفي عام 
وبمعدل نمو سنوي معنوي إحصائيا عند مستوى 0.05 قدر بنحو 1.70\%

وبإجراء اختبار t بين متوسطي فترتي الدراسة للتعرف على وجود اختلافات بينهما، تبين أن قيمة t المحسوبة بلغت فئت 9.49 وهي معنوية عند مستوى 0.05، وهذا بعني أنه يمكن رفض الفرض الصفري Ho القائل بأنه لا يوجد فروق بين منوسطي الإنتاج الكلي للعنب قبل وبعد نطبيق نظام التبع، وقبول الفرض البديل H بوجود فروق معنوية بين منوسط

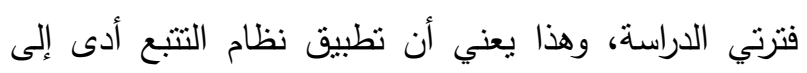
زيادة الإنتاج الكلي للعنب.

\section{تطور إنتاجية محصول العنب المصري:}

بدراسة تطور إنتاجية محصول العنب في جمهورية مصر

العربية خلال الفترة 1994-2019، أتضح من الجدول (1) أنها تتراوح بين حد أدنى بلغ حوالي 5.80 طن/ فدان عام1994، وحد أقصى بلغ حوالي 9.58 طن/ فدان عام لـن 2008 وبمنوسط سنوي بلغ حوالي 8.23 طن/ فدان. وتتير بيانات الجدول (2) أن إنتاجية محصول العنب اتجهت للزيادة خلال الفترة الأولى بمقدار زيادة سنوي معنوي بلغ حوالي 0.26 طن/ فدان وبمعامل اختلاف بلغ نحو 14.85\% وهو ما يشير إلى عدم وجود تشتت في إنتاجية محصول العنب خلال تلك الفترة، وبمعدل نمو سنوي معنوي إحصائيا عند مستوى 0.05 قدر بنحو 3.40\%. بينما اتجهت إنتاجية محصول العنب للانخفاض خلال الفترة الثانية بمقدار انخفاض سنوي معنوي بلغ حوالي 0.015 طن/ فدان

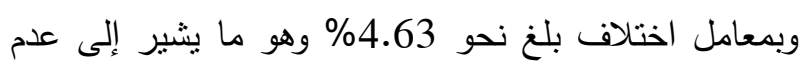
وجود تشتت في إنتاجية محصول العنب خلال نلاك الفترة، وبمعدل نتاقص سنوي معنوي إحصائيا عند مستوى 0.05 قدر بنحو 0.2\% وبإجراء اختبار t بين منوسطي فترتي الدراسة للتعرف على وجود اختلافات بينهما، تبين أن قيمة t المحسوبة بلغت 3.56 وهي معنوية عند مستوى 0.05،
محصول العنب اتجهت للزيادة خلال الفترة الثانية بمقدار زيادة سنوي معنوي بلغ حوالي 3.21 ألف فدان، وبمعامل اختلاف بلغ نحو 8.04\% وهو ما يشير إلى عدم وجود تشنت في مساحة محصول العنب خلال تلك الفترة، وبمعدل نمو سنوي معنوي إحصائيا عند مستوى 0.05 قدر بنحو .\% 1.90 وبإجراء اختبار t بين متوسطي فترتي الدراسة للتعرف

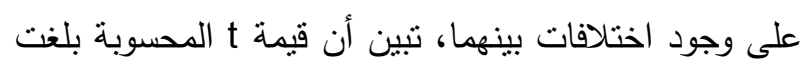
12.27 وهي معنوية عند مسنوى 0.05، وهذا يعني أنه يمكن رفض الفرض الصفري H القائل بأنه لا يوجد فروق بين متوسطي مساحة محصول العنب قبل وبعد تطبيق نظام

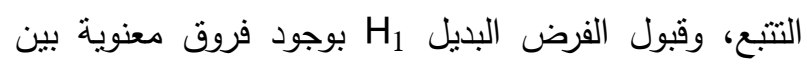
المتوسطات، وهذا يعني أن تطبيق نظام التتبع أدى إلى زيادة مساحة محصول العنب. تطور الإنتاج الكلي لمحصول العنب المصري: بدراسة تطور الإنتاج الكلي لمحصول العنب في جمهورية مصر العربية خلال الفترة 1994-2019، أتضح من جدول (1) أنها تراوحت بين حد أدنى بلغ حوالي 707.05 ألف طن من جن عام 1994، وحد أقصى بلغ حوالي 1734.42 ألف طن بن سلن عام 2017 وبمنوسط سنوي بلغ حوالي 1292.54 ألف طن. وتثنير بيانات الجدول (2) أن الإنتاج الكلي لمحصول العنب اتجه للزيادة خلال الفترة الأولى بمقدار زيادة سنوي

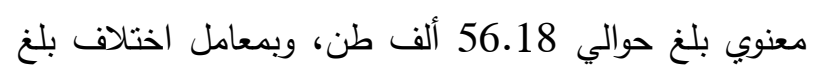
نحو 21.23\% وهو ما يشير إلى عدم وجود تشتت في الإنتاج الكلي للعنب خلال تلك الفترة، وبمعدل نمو سنوي معنوي إحصائيا عند مستوى 0.05 قدر بنحو 5.4\%، كما

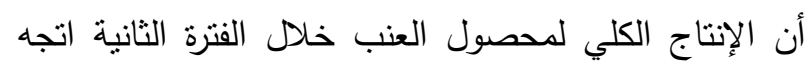

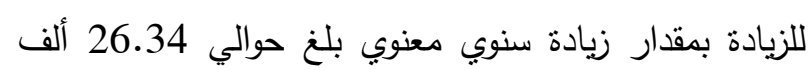

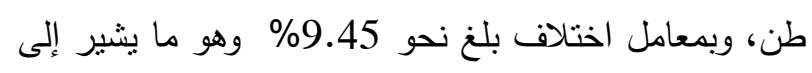
عدم وجود تشنت في الإنتاج الكلي للعنب خلال تلك الفترة، 
أحمد أبو اليزيد الرسول، سامح محمد حسن شهاب واخرون...: تأثير تطبيق نظام التتبع على صادرات مصر من العنب.

جدول 1. تطور مساحة وانتاج وانتاجية العنب في جمهورية مصر العربية خلال القترة 1994- 2019

\begin{tabular}{|c|c|c|c|}
\hline 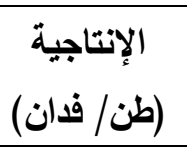 & الإنتاج الكي & (ألف فاحة) & السنة \\
\hline 5.80 & 707.05 & 121.90 & 1994 \\
\hline 6.08 & 739.48 & 121.54 & 1995 \\
\hline 7.64 & 943.70 & 123.46 & 1996 \\
\hline 6.94 & 867.91 & 125.01 & 1997 \\
\hline 7.43 & 957.73 & 128.93 & 1998 \\
\hline 6.88 & 1009.56 & 146.64 & 1999 \\
\hline 7.28 & 1075.10 & 147.69 & 2000 \\
\hline 7.00 & 1078.91 & 154.09 & 2001 \\
\hline 7.72 & 1073.82 & 139.02 & 2002 \\
\hline 8.47 & 1196.85 & 141.38 & 2003 \\
\hline 8.87 & 1275.29 & 143.80 & 2004 \\
\hline 9.27 & 1391.75 & 150.09 & 2005 \\
\hline 9.37 & 1431.97 & 152.84 & 2006 \\
\hline 9.58 & 1485.01 & 155.01 & 2007 \\
\hline 9.58 & 1531.42 & 159.78 & 2008 \\
\hline 8.67 & 1370.24 & 158.05 & 2009 \\
\hline 8.60 & 1360.25 & 158.23 & 2010 \\
\hline 8.24 & 1320.80 & 160.21 & 2011 \\
\hline 8.42 & 1378.82 & 163.74 & 2012 \\
\hline 8.41 & 1434.67 & 170.53 & 2013 \\
\hline 8.95 & 1596.17 & 178.39 & 2014 \\
\hline 9.11 & 1686.71 & 185.15 & 2015 \\
\hline 8.84 & 1691.19 & 191.31 & 2016 \\
\hline 8.97 & 1734.42 & 193.28 & 2017 \\
\hline 8.91 & 1641.08 & 184.17 & 2018 \\
\hline 8.97 & 1626.26 & 181.26 & 2019 \\
\hline 8.23 & 1292.54 & 155.21 & المتوسط \\
\hline
\end{tabular}


جدول 2. معادلات الاتجاه الزمني للمتغيرات الإنتاجية للعنب خلال الفترة 1994- 2019

\begin{tabular}{|c|c|c|c|c|c|c|c|}
\hline السنول التغير & المتوسط & الاختلاف \% معل & $\mathbf{F}$ & $\mathbf{R}^{2}$ & 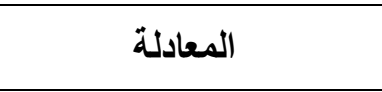 & المتغير & 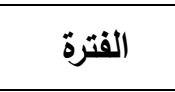 \\
\hline $2.00^{\circ}$ & 138.18 & 8.94 & $26.92^{\circ}$ & 0.71 & $\begin{array}{c}\hat{\mathrm{Y}}_{\mathrm{Y}}=119.47+2.67 \mathrm{X}_{\mathrm{t}} \\
(29.21)^{\circ}(5.19)^{\circ}\end{array}$ & بالألف فدانة & \multirow{3}{*}{ الفتبل تطبيقي الأولى } \\
\hline $5.40^{\circ}$ & 1057.62 & 21.23 & $207.76^{\circ}$ & 0.95 & $\begin{array}{c}\hat{\mathrm{Y}}_{\mathrm{t}}=664.33+56.18 \mathrm{X}_{\mathrm{t}} \\
(21.47)^{\circ} \quad(14.41)^{\circ}\end{array}$ & بألألَّاج طن الكلي & \\
\hline $3.40^{\circ}$ & 7.60 & 14.85 & $47.75^{\circ}$ & 0.81 & $\begin{array}{l}\hat{Y}_{t}=5.77+0.26 X_{t} \\
(19.23)^{\circ}(6.91)^{\circ}\end{array}$ & طالإنتاجية فدان & \\
\hline $1.90^{\circ}$ & 172.24 & 8.04 & $48.03^{\circ}$ & 0.81 & $\begin{array}{c}\hat{\mathrm{Y}}_{\mathrm{Y}}=149.77+3.21 \mathrm{X}_{\mathrm{t}} \\
(40.75)^{\circ}(6.93)^{\circ}\end{array}$ & بالألف فدانة & \multirow{3}{*}{ 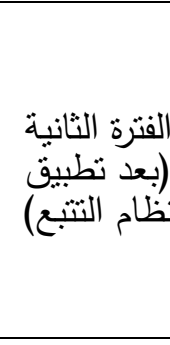 } \\
\hline $1.70^{\circ}$ & 1527.46 & 9.45 & $11.21^{* *}$ & 0.51 & $\begin{aligned} \hat{Y}_{t} & =1343.06+26.34 X_{t} \\
& (21.50)^{\circ} \quad(3.35)^{\circ}\end{aligned}$ & بألألتَّاج طن الكلي & \\
\hline \multirow[t]{5}{*}{$(0.20)^{*}$} & 8.87 & 4.63 & $0.24^{\text {ns }}$ & 0.02 & $\begin{array}{c}\hat{Y}_{t}=8.97-0.015 X_{t} \\
(35.99)^{\circ}(-0.49)^{n s}\end{array}$ & طن الإنتاجية فدان & \\
\hline & & & \multicolumn{5}{|c|}{ 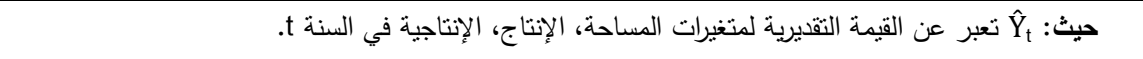 } \\
\hline & & & \multicolumn{3}{|c|}{ t تعبر عن السنوات= (13)........ } & \multicolumn{2}{|c|}{ تعبر عن متغير الزمن في السنة t. } \\
\hline & & & \multirow{2}{*}{\multicolumn{2}{|c|}{ 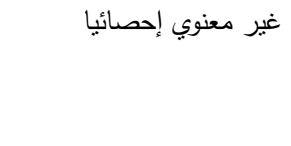 }} & معنوي عند مستوى 0.01 & 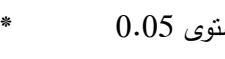 & 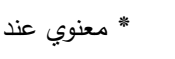 \\
\hline & & & & & التخير السنوي السالب. & لأوفواس تنل على معدل & ( ) الأرقام بي \\
\hline
\end{tabular}

تنشير بيانات الجدول (4) أن كمية صادرات العنب اتجهت للزيادة خلال الفترة الأولى بمقدار زيادة سنوي معنوي بلغ حوالي 2.01 ألف طن وبمعامل اختلاف بلغ نحو لهن 125.20 وهو ما بثير إلى وجود نتشت كبير جدا في كمية صادرات العنب خلال تلاك الفترة، وبمعدل نمو سنوي معنوي إحصائيا عند مستوى 0.05 قدر بنحو 30.8 \% من من كند منوسط كمية الصادرات من العنب خلال الفترة الأولى. كما اتجهت كمية صلادرات العنب للزيادة خلال الفترة الثانية بمقار زيادة سنوي معنوي بلغ حوالي 1.44 ألف طن

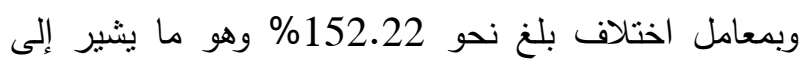
وجود نتشت كبير جدا في كمية صادرات العنب خلال نلك إلك

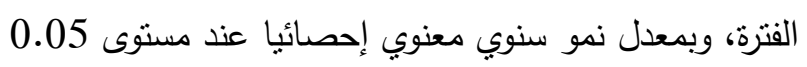

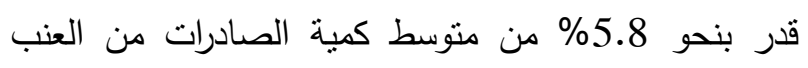

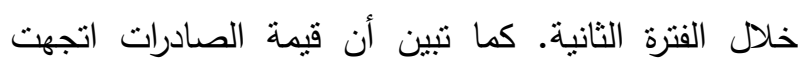

وهذا يعني أنه يمكن رفض الفرض الصفري H القائل بأنه لا

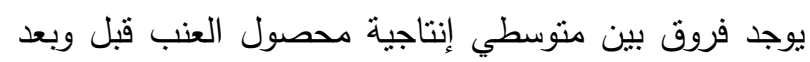

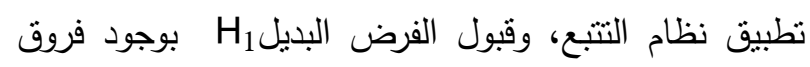

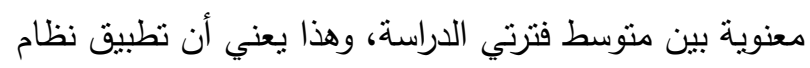
التتبع أدى إلى زيادة إنتاجية محصول العنب. 2- تطور كمية وقيمة صادرات العنب: تطور كمية وقيمة صادرات العنب لدول العالم:

بدراسة تطور كمية وقيمة الصادرات المصرية من العنب

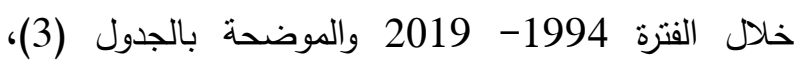
أنضح أن كمية الصادرات المصرية من العنب تراوحت بين حد أدنى بلغ نحو 0.78 ألف طن عام 1998 وحد أقصى الصى بلغ نحو 154.21 ألف طن عام 2019 وبمتوسط بلغ 48.79 ألف طن خلال الفترة محل الدراسة. 

أحمد أبو اليزيد الرسول، سامح محمد حسن شهاب واخرون...: تأثنر تطبيق نظام التتبع على صادرات مصر من العنب.

للزيادة خلد الفترة الأولى بمقدار زيادة سنوي بلغ حوالي بمكن رفض الفرض الصفري Ho القائل بأنه لا يوجد فروق 4.67 مليون دولار وبمعامل اختلاف بلغ نحو 35.64\% بين منوسطي كمية الصادرات المصرية من العنب لدول بهات وهو ما يشير إلى عدم وجود تشتت في قيمة الصادرات خلال العالم قبل وبعد تطبيق نظام التتبع، وقبول الفرض البديل

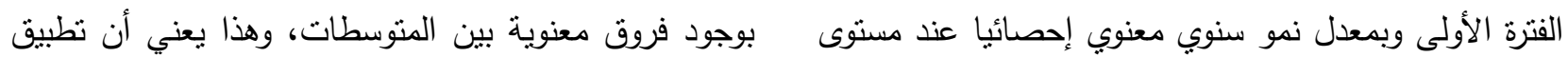

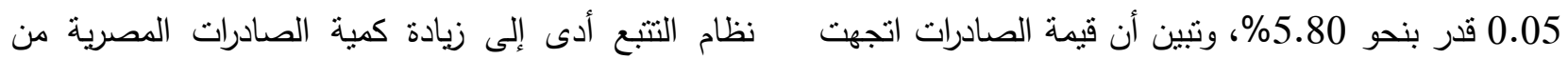

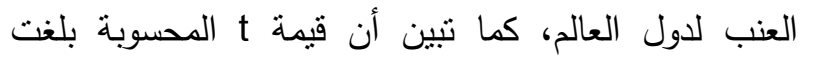
11.59 وهي معنوية عند مستوى 0.05، وهذا يعني أنه للزيادة خلال الفترة الثانية بمقدار زيادة سنوي بلغ حوالي 9.89 مليون دولار وبمعامل اختلاف بلغ نحو 32.14 وهو ما يشير إلى عدم وجود تشتت في قيمة الصادرات خلال بمكن رفض الفرض الصفري H القائل بأنه لا يوجد فروق الفترة الثانية وبمعدل نمو سنوي معنوي إحصائيا عند مسنوى بين منوسطي قيمة الصادرات المصرية من العنب لدول العالم قبل وبعد تطبيق نظام التتبع، وقبول الفرض البديل بوجود 0.05 قدر بنحو 7.4\% فروق معنوية بين المتوسطات، وهذا يعني أن نطبيق نظام

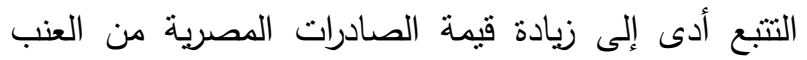

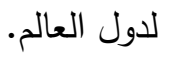

وبإجراء اختبار t بين منتوسط فترتي الدراسة للتعرف على وجود اختلافات بينهما، تبين أن قيمة t المحسوبة بلغت 10.72 وهي معنوية عند مستوى 0.05، وهذا بعني أنه

جدول3. تطور كمية وقيمة الصادرات المصرية من العنب لدول العالم خلال الفترة 1994- 2019

\begin{tabular}{|c|c|c|}
\hline القيمة (مليون دولار) & الكمية (ألف طن) & السنة \\
\hline 0.61 & 1.27 & 1994 \\
\hline 0.47 & 1.14 & 1995 \\
\hline 0.91 & 1.30 & 1996 \\
\hline 0.50 & 0.83 & 1997 \\
\hline 0.51 & 0.78 & 1998 \\
\hline 0.45 & 0.89 & 1999 \\
\hline 1.88 & 4.59 & 2000 \\
\hline 1.29 & 4.55 & 2001 \\
\hline 1.82 & 5.99 & 2002 \\
\hline 2.93 & 7.42 & 2003 \\
\hline 11.44 & 15.35 & 2004 \\
\hline 16.83 & 24.60 & 2005 \\
\hline 21.92 & 27.81 & 2006 \\
\hline 59.69 & 53.70 & 2007 \\
\hline 91.93 & 49.74 & 2008 \\
\hline 225.38 & 135.59 & 2009 \\
\hline 115.01 & 52.86 & 2010 \\
\hline 210.06 & 62.33 & 2011 \\
\hline 224.31 & 116.05 & 2012 \\
\hline 183.36 & 88.14 & 2013 \\
\hline 157.43 & 79.20 & 2014 \\
\hline 182.00 & 84.37 & 2015 \\
\hline 179.41 & 93.35 & 2016 \\
\hline 232.96 & 103.54 & 2017 \\
\hline 221.55 & 99.00 & 2018 \\
\hline 234.89 & 154.21 & 2019 \\
\hline 91.52 & 48.79 & المتوسط \\
\hline
\end{tabular}




\begin{tabular}{|c|c|c|c|c|c|c|c|}
\hline التسنير \% & المتوسط & الاختلاف \% & $\mathbf{F}$ & $\mathbf{R}^{2}$ & المعادلة & المتغير & الفترة \\
\hline $30.80^{\circ}$ & 7.42 & 125.20 & $27.19^{\prime \prime}$ & 0.71 & $\begin{array}{c}\hat{Y}_{\mathrm{t}}=-6.67+2.01 \mathrm{X}_{\mathrm{t}} \\
(-2.18)^{\mathrm{ns}}(5.21)^{*}\end{array}$ & بالألفادرات طن & الفترة الأولىي \\
\hline $5.80^{\circ}$ & 90.16 & 35.64 & $5.18^{\circ}$ & 0.32 & $\begin{array}{l}\hat{\mathrm{Y}}_{\mathrm{t}}=57.47+4.67 \mathrm{X}_{\mathrm{t}} \\
(2.28)^{*}\end{array}$ & بالمليون دولاتر & نظام التتبع) \\
\hline $32.10^{\circ}$ & 4.73 & 152.22 & $17.10^{\prime \prime}$ & 0.61 & $\begin{array}{c}\hat{\mathrm{Y}}_{\mathrm{t}}=-5.36+1.44 \mathrm{X}_{\mathrm{t}} \\
(-1.94)^{\mathrm{ns}}(4.13)^{*}\end{array}$ & بالألفادرات طن & الفتزة الثانية \\
\hline $7.40^{\circ}$ & 178.30 & 32.14 & $9.06^{\circ}$ & 0.45 & $\begin{array}{c}\hat{\mathrm{Y}}_{\mathrm{t}}=109.08+9.89 \mathrm{X}_{\mathrm{t}} \\
\quad(4.18)^{*} \quad(3.01)^{*}\end{array}$ & بالمليون دولاتر & نظام التنبع) \\
\hline
\end{tabular}

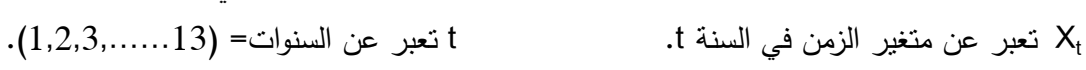

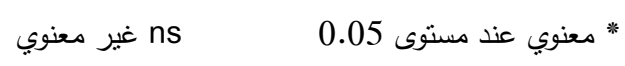

المصدر : جمعت وحسبت من بيانات الجدول (4).

مستوى0.05 قدر بنحو 38\%، بينما اتجهت كمية صادرات

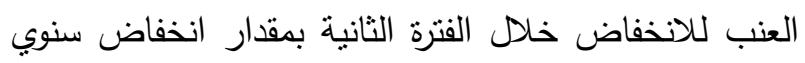

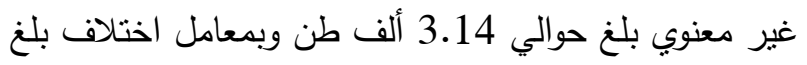
نحو 49.83\% وهو ما بشير إلى عدم وجود نشتت في كمية صادرات العنب وبمعدل تناقص سنوي غير معنوي قدر بنحو 2.10\%. كما ثبين أن قيمة صادرات العنب اتجهت للزيادة خلال الفترة الأولى بمقدار زيادة سنوي معنوي بلغ بلغ حوالي

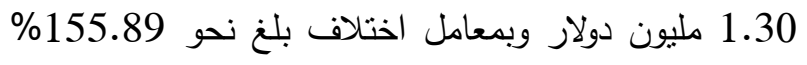

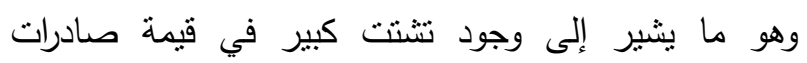
العنب، وبمعدل نمو سنوي معنوي إحصائيا عند مستوى

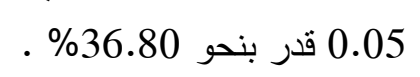

تطور كمية وقيمة صادرات مصر من العب لاول الاتحاد الأورويي: بدراسة نطور كمية وقيمة صادرات مصر من العنب خلال الفترة 1994- 2019 والموضحة بالجدول (5) (5)، يتضح أن كمية الصادرات المصرية من العنب لدول الاتحاد

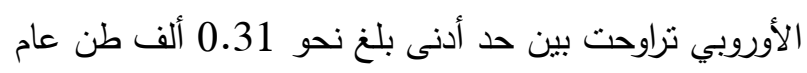
1998 وحد أقصى بلخ نحو 184.67 ألف طن عام 2008 وبمتوسط بلغ 41.41 ألف طن خلال الفترة محل الدراسة. وتتشير بيانات الجدول (6) أن كمية صادرات مصر من العنب اتجهت للزيادة خلال الفترة الأولى بمقدار زيادة سنوي التيات

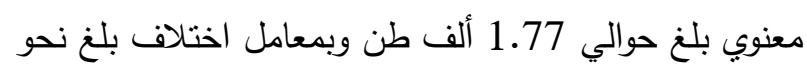
134.39 وهو ما يثير إلى وجود نتشت كبير في كمية صادرات العنب، وبمعدل نمو سنوي معنوي إحصائيا عند 
جلول5. تطور كمية وقيمة الصادرات المصرية من العنب لاول الاتحاد الأورويي خلال الفترة 1994 - 2019

\begin{tabular}{|c|c|c|}
\hline القيمة بالمليون دولار & الكمية بالألف طن & السنة: \\
\hline 0.21 & 0.53 & 1994 \\
\hline 0.24 & 0.52 & 1995 \\
\hline 0.75 & 0.57 & 1996 \\
\hline 0.54 & 0.43 & 1997 \\
\hline 0.34 & 0.31 & 1998 \\
\hline 0.35 & 0.51 & 1999 \\
\hline 1.64 & 3.43 & 2000 \\
\hline 1.14 & 3.64 & 2001 \\
\hline 1.62 & 5.03 & 2002 \\
\hline 2.28 & 5.76 & 2003 \\
\hline 10.05 & 12.91 & 2004 \\
\hline 15.35 & 21.97 & 2005 \\
\hline 19.31 & 23.39 & 2006 \\
\hline 52.10 & 42.41 & 2007 \\
\hline 147.09 & 184.67 & 2008 \\
\hline 193.51 & 116.89 & 2009 \\
\hline 170.59 & 68.70 & 2010 \\
\hline 161.90 & 48.18 & 2011 \\
\hline 171.63 & 89.06 & 2012 \\
\hline 135.41 & 64.34 & 2013 \\
\hline 111.63 & 51.85 & 2014 \\
\hline 124.23 & 48.93 & 2015 \\
\hline 122.63 & 57.06 & 2016 \\
\hline 156.48 & 69.54 & 2017 \\
\hline 159.38 & 71.30 & 2018 \\
\hline 158.61 & 84.86 & 2019 \\
\hline 73.81 & 41.41 & المتوسط \\
\hline
\end{tabular}

المصدر : جمعت وحسبت من - خريطة التجارة التابعة للأمم المتحدة www.trade.map.org www.fao.org بيانات منظمة الأغذية والزراعة العالمية (الفاو) -

بوجود فروق معنوية بين المتوسطات، وهذا يعني أن تطبيق نظام التتبع أدى إلى زيادة كمية الصادرات المصرية

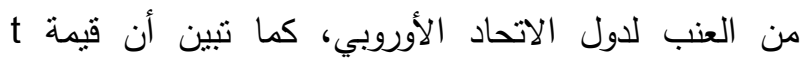
المحسوبة بلغت 14.41 وهي معنوية عند مستوى 0.05، وهذا يعني أنه يمكن رفض الفرض الصفري Ho القائل بأنه لا يوجد فروق بين متوسطي قيمة الصادرات المصرية من العنب لدول الاتحاد الأوروبي قبل وبعد تطبيق نظام التتبع، وقبول الفرض البديل بوجود فروق معنوية بين المتوسطات، وهذا يعني أن نطبيث نظام التبع أدى إلى زيادة قيمة الصادرات

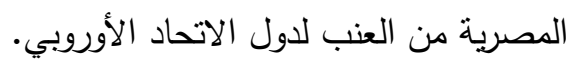

كما تبين أن قيمة صادرات العنب اتجهت للزيادة خلال الفترة الثانية بمقدار زيادة سنوي غير معنوي بلغ حوالي 1.50

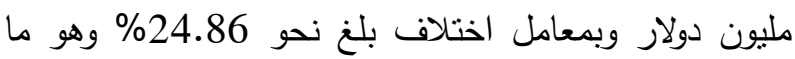
يشير إلى عدم وجود نتنت في قيمة صادرات العنب وبمعدل نمو سنوي غبر معنوي قدر بنحو 2.40\%. وبإجراء اختبار t بين منوسط فترتي الدراسة للتعرف على وجود اختلافات بينهما، تبين أن قيمة t المحسوبة بلغت 6.39 وهي معنوية عند مستوى 0.05، وهذا يعني أنه يمكن رفض الفرض الصفري Ho القائل بأنه لا يوجد فروق بين متوسطي كمية الصادرات المصرية من العنب لدول الاتحاد الأوروبي قبل وبعد تطبيق نظام النتبع، وقبول الفرض البديل 
جدول6. معادلات الاتجاه الزمني لكمية وقيمة الصادرات المصرية من العب لدول الاتحاد الأوروبي خلال الفترة 19942019

\begin{tabular}{|c|c|c|c|c|c|c|c|}
\hline السنوي التغير & المتوسط & الاختلاف & $\mathbf{F}$ & $\mathbf{R}^{2}$ & المعادلة & المتغير & الفترة \\
\hline $38.00^{\circ}$ & 6.08 & 134.39 & $27.24^{\circ}$ & 0.71 & $\begin{array}{c}\hat{Y}_{t}=-6.32+1.77 X_{t} \\
\quad(-2.35)^{\circ} \quad(5.22)^{\circ}\end{array}$ & بالمليةن دولادرات & القترة الأولى \\
\hline 36.8 & 4.14 & 155.89 & $17.36^{*}$ & 0.61 & $\begin{array}{c}\hat{\mathrm{Y}}_{\mathrm{Y}}=-4.94+1.30 \mathrm{X}_{\mathrm{t}} \\
(-2.00)^{\mathrm{ns}}(4.17)^{\circ}\end{array}$ & بأتمليون دولار & نظام التتبع) \\
\hline$(2.10)^{\mathrm{ns}}$ & 76.75 & 49.83 & $1.26^{\mathrm{ns}}$ & 0.10 & $\begin{aligned} \hat{\mathrm{Y}}_{\mathrm{t}} & =98.76-3.14 \mathrm{X}_{\mathrm{t}} \\
& (4.43)^{\circ} \quad(-1.12)^{\mathrm{ns}}\end{aligned}$ & كبالملية الصادرات دولار & الفتدرة الثانية \\
\hline $2.40^{\mathrm{ns}}$ & 143.48 & 24.86 & ${ }^{\mathrm{n} s} 0.30$ & 0.03 & $\begin{array}{c}\hat{\mathrm{Y}}_{\mathrm{t}}=132.97+1.50 \mathrm{X}_{\mathrm{t}} \\
(6.15)^{\circ} \quad(0.55)^{\mathrm{ns}}\end{array}$ & بآثمليون الصادرات & نظّام التتبع) \\
\hline
\end{tabular}

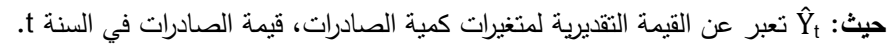

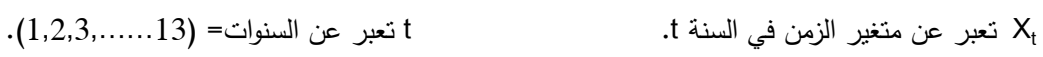

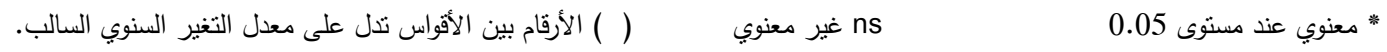
المصدر : جمعت وحسبت من بيانات الجدول (6).

صادرات مصر من العنب كمنوسط للفترة 1994- 2019، الأمر الذي يشير إلى وجود نركز كبير في أسواق التصدير بين عدد من دول الاتحاد الأوروبي. التوزيع الجغرافي لصادرات العنب لدول الاتحاد الأوروبي: بدراسة التوزيع الجغرافي للصادرات الصصرية من العنب كمتوسط للفترة 1994- 2019 والموضح في الجدول (8)، الجنران يتضح أن إجمالي الكمية المصدرة إلى دول الاتحاد الأوروبي 41.41 ألف طن يمثل نحو 84.87\% من إجمالي الكمية

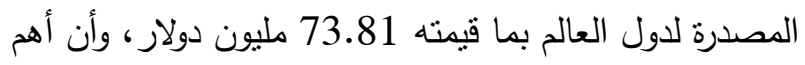
أسواق تصدير العنب المصري لدول الاتحاد الأوروبي تمنتل

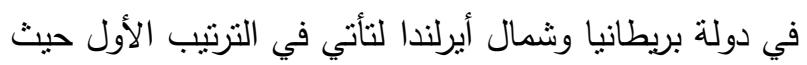
بلغت الكمية الدصدرة 27.74 ألف طن بما قيمته 34.71 مليون دولار والتي تمنل حوالي 66.99\% من الكمية

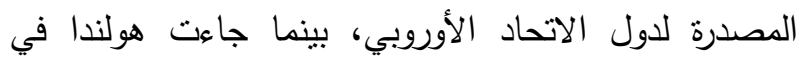
المركز الثاني حيث بلغت الكمية الصصدرة 15.86 ألف طن لأن الترون
3-التوزيع الجغرافي لصادرات العنب التوزيع الجغرافي لصادرات العنب لدول العالم: بدراسة التوزيع الجغرافي للصادرات الدصرية من العنب

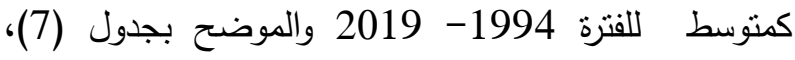
أتضح أن إجمالي الكمية المصدرة من العنب المصري قد بلغت نحو 48.79 ألف طن، بما قيمته 91.51 مليون دولار وأن إجمالي الكمية الدصدرة إلى دول الاتحاد الأوروبي 41.41 ألف طن يمتل نحو 84.87\% من إجمالي الكمية

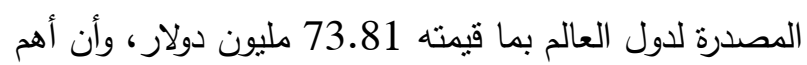
أسواق تصدير العنب المصري تنتلت في بريطانيا وشمال

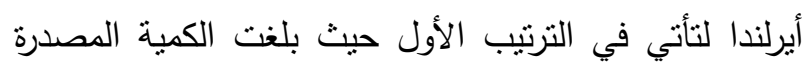

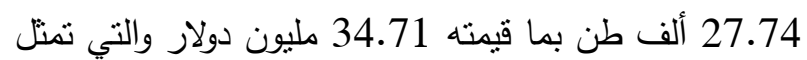
ثلث الكمية المصدرة لدول العالم، بينما جاءت هولندا والمانيا

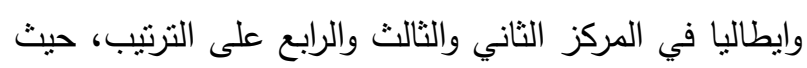
تمثل نلالك الأسواق مجتمعة نحو 34.73\% من إجمالي 
بما قيمته 22.22 مليون دولار تمنل حوالي 38.29\% من تللك الأسواق مجتمعة نحو 58.31\% من إجمالي كمية الكمية المصدرة لدول الاتحاد الأوروبي، وجاءت ألمانيا صادرات مصر من العنب لدول الاتحاد الأوروبي كمتوسط وايطاليا وبلجيكا وسلوفينيا وفرنسا وايرلندا والدنمارك وبولندا للفترة 1994- 2019. في المركز الثالث وحتى العاشر على الترنيب، حيث تمنل

\begin{tabular}{|c|c|c|c|c|}
\hline$\%$ & قيمة الصادرات بالمليون & $\%$ & كمية الصادرات بالألف & الدولة الدئ \\
\hline 71.15 & 34.71 & 30.31 & 27.74 & بريطانيا وشمال ايرلندا \\
\hline 45.53 & 22.22 & 17.33 & 15.86 & هولندا - \\
\hline 21.11 & 10.30 & 8.78 & 8.04 & إلمانيا \\
\hline 17.15 & 8.37 & 8.62 & 7.89 & إيطاليا \\
\hline 16.87 & 8.23 & 8.00 & 7.32 & روسياً الاتحادية \\
\hline 8.42 & 4.11 & 5.05 & 4.62 & بلجيكا \\
\hline 7.79 & 3.80 & 3.75 & 3.43 & الإمارات العربية المتحدة \\
\hline 5.49 & 2.68 & 2.75 & 2.51 & سلوفينيا \\
\hline 4.84 & 2.36 & 2.69 & 2.46 & السعودية \\
\hline 1.62 & 0.79 & 2.16 & 1.97 & السودان \\
\hline 80.65 & 73.81 & 84.87 & 41.41 & إجمالى دول الاتحاد الأوروبى \\
\hline 100 & 91.52 & 100 & 48.79 & إجمالى دول العالم \\
\hline
\end{tabular}

جدول 8. التوزيع الجغرافي للصادرات المصرية من العنب لدول الاتحاد الأورويي كمتوسط للفترة 1994- 2019

\begin{tabular}{|c|c|c|c|c|}
\hline$\%$ & قيمة الصادرات بالمليون & $\%$ & كمية الصادرات بالألف & 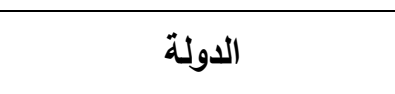 \\
\hline 47.03 & 34.71 & 66.99 & 27.74 & بريطانيا وشمال أيرلندا \\
\hline 30.10 & 22.22 & 38.29 & 15.86 & هولندا \\
\hline 13.95 & 10.30 & 19.41 & 8.04 & آلمانبا \\
\hline 11.33 & 8.37 & 19.06 & 7.89 & إيطاليا \\
\hline 5.56 & 4.11 & 11.15 & 4.62 & بلجيكًا \\
\hline 3.63 & 2.68 & 6.07 & 2.51 & سلوفينيا \\
\hline 0.56 & 0.41 & 0.78 & 0.32 & فرنسا \\
\hline 0.30 & 0.23 & 0.65 & 0.27 & ايرلندا \\
\hline 0.32 & 0.23 & 0.61 & 0.25 & الدنمارك \\
\hline 0.20 & 0.15 & 0.58 & 0.24 & بولندا \\
\hline 80.65 & 73.81 & 84.87 & 41.41 & إجمالى دول الاتحاد الأوروبى \\
\hline 100 & 91.52 & 100 & 48.79 & إجمالى دول العالم \\
\hline
\end{tabular}




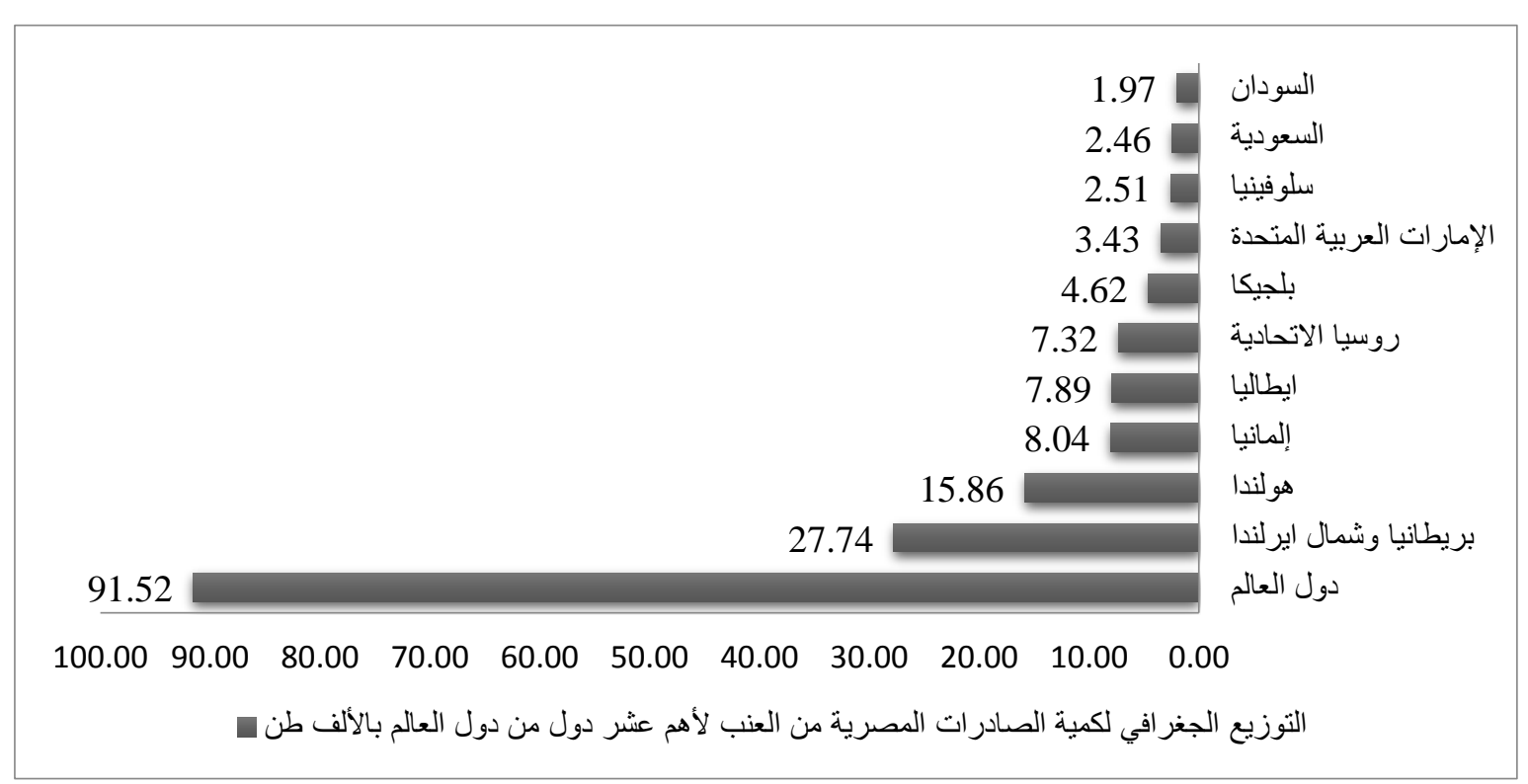

الثكل رقم 1. التوزيع الجغرافي لكمية الصادرات المصرية من العنب لأهم عثر دول من دول العالم بالألف طن

المصدر : بيانات جدول 7.

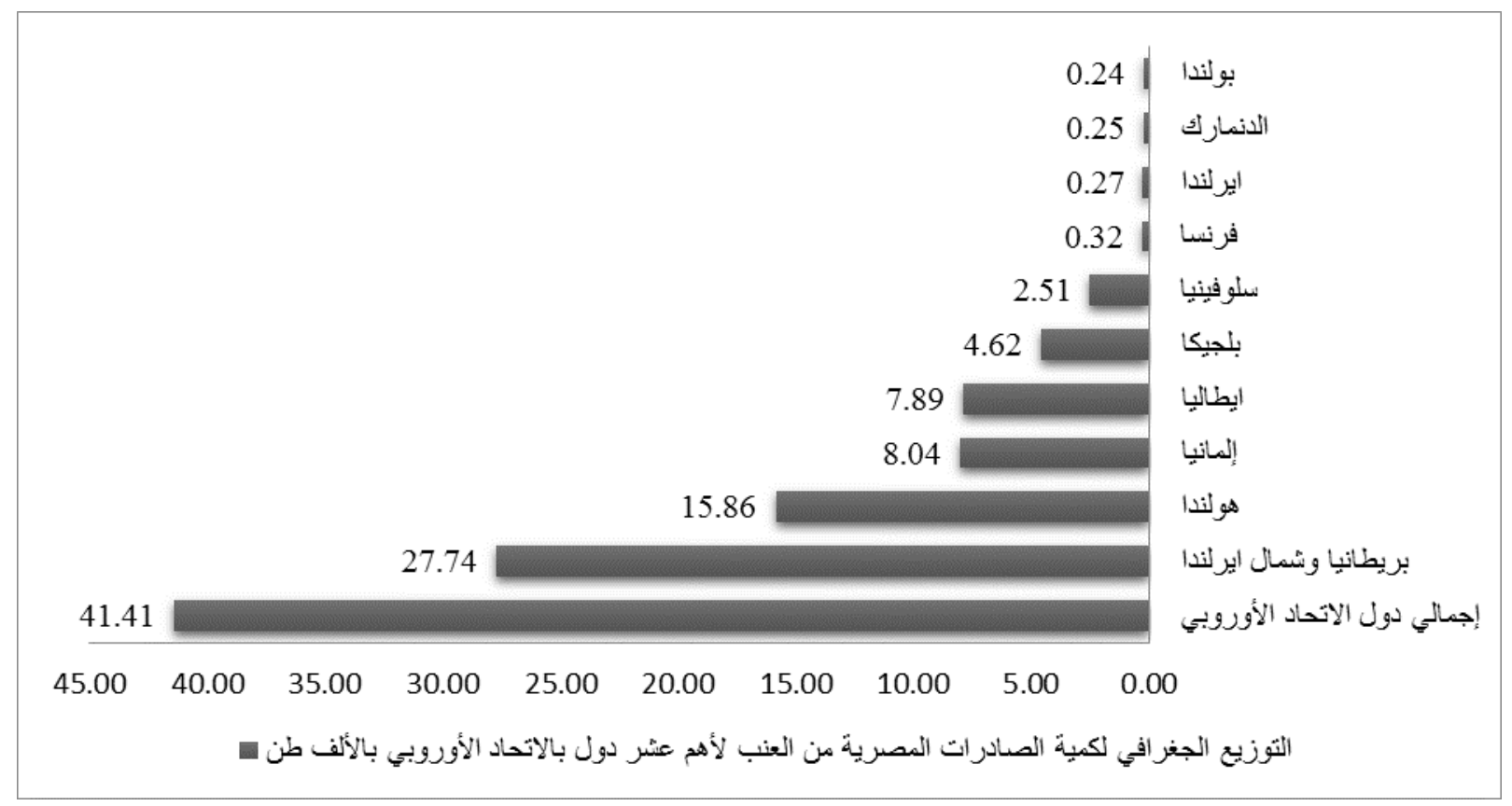

الثكل رقم 2. التوزيع الجغرافي لكمية الصادرات المصرية من العنب لأهم عثر دول بالاتحاد الأوروبي بالألف طن 
الجدول تذبذب قيمة معامل الميزة النسبية الظاهرة بين الارتفاع عدة أعوام ثم الانخفاض ثم الارتفاع مرة أخرى وبلغ متوسط معامل الميزة النسبية الظاهرة حوالي 4.14 خلا الفترة 1994-2019، الأمر الذي بعني أن هذا المحصول التصديري من المحاصيل الهامة التي تتافس بشدة في الأسواق العالمية بشرط المحافظة على الأسواق التصديرية لهذا المحصول وذلك من خلا مطابقة المواصفات المطلوبة للسوق العالمي وفتح أسواق جديدة تستوعب إنتاج مصر من

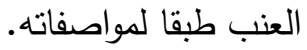

4- أهم مؤثرات قياس القدرة التنافية لصادرات مصر من

Revealed مؤشر الميزة النسبية Comparative Advantage Index

يوضح الجدول (9) مؤشر الميزة النسبية الظاهرة لمحصول العنب خلال الفترة 1994- 2019 حيث أتضح أنه على الرغم من أن قيمة المؤشر نزيد عن الواحد الصحيح خلال تلك الفترة مما يعني وجود ميزة نسبية ظاهرة لصادرات مصر من العنب في الأسواق الخارجية، إلا أنه تبين من نفس

جدول9. تطور قيمة الصادرات الزراعية المصرية والعالمية خلال الفترة 1994- 2019

\begin{tabular}{|c|c|c|c|c|c|}
\hline \multirow{2}{*}{ الميزة النسبية } & \multicolumn{2}{|c|}{ الصادرات العالمية (مليون دولار) } & \multicolumn{2}{|c|}{ الصادرات المصرية (مليون دولار) } & \multirow{2}{*}{ 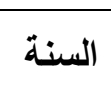 } \\
\hline & الصادرات الزراعية & العنب & الصادرات الزراعيةه & العنب & \\
\hline 0.23 & 388443.63 & 1882.39 & 551.51 & 0.61 & 1994 \\
\hline 0.20 & 442957.50 & 1951.52 & 536.12 & 0.47 & 1995 \\
\hline 0.39 & 465626.80 & 2069.83 & 521.09 & 0.91 & 1996 \\
\hline 0.23 & 457665.53 & 2271.34 & 442.25 & 0.50 & 1997 \\
\hline 0.18 & 437782.59 & 2194.22 & 571.78 & 0.51 & 1998 \\
\hline 0.14 & 417229.21 & 2298.37 & 585.76 & 0.45 & 1999 \\
\hline 0.59 & 411161.40 & 2502.36 & 518.14 & 1.88 & 2000 \\
\hline 0.35 & 414533.40 & 2493.13 & 620.49 & 1.29 & 2001 \\
\hline 0.39 & 442861.56 & 2693.66 & 771.78 & 1.82 & 2002 \\
\hline 0.52 & 525543.77 & 3135.46 & 937.75 & 2.93 & 2003 \\
\hline 1.61 & 607641.30 & 3292.70 & 1314.30 & 11.44 & 2004 \\
\hline 2.38 & 653805.37 & 3956.87 & 1167.54 & 16.83 & 2005 \\
\hline 3.65 & 721854.24 & 3987.60 & 1086.38 & 21.92 & 2006 \\
\hline 6.76 & 873724.96 & 4933.99 & 1563.41 & 59.69 & 2007 \\
\hline 8.03 & 1068026.48 & 5615.86 & 2176.84 & 91.93 & 2008 \\
\hline 8.60 & 951564.82 & 5659.59 & 4407.02 & 225.38 & 2009 \\
\hline 6.88 & 1085628.37 & 6215.58 & 2918.03 & 115.01 & 2010 \\
\hline 7.80 & 1321445.18 & 6987.10 & 5093.77 & 210.06 & 2011 \\
\hline 10.06 & 1338957.94 & 7211.49 & 4140.79 & 224.31 & 2012 \\
\hline 6.67 & 1397951.12 & 7893.88 & 4867.51 & 183.36 & 2013 \\
\hline 6.44 & 1421836.11 & 7912.72 & 4395.77 & 157.43 & 2014 \\
\hline 6.81 & 1275064.06 & 7786.79 & 4378.14 & 182.00 & 2015 \\
\hline 6.76 & 1287315.82 & 7842.76 & 4354.92 & 179.41 & 2016 \\
\hline 7.76 & 1411289.24 & 8603.07 & 4921.54 & 232.96 & 2017 \\
\hline 7.35 & 1454098.53 & 8742.93 & 5013.69 & 221.55 & 2018 \\
\hline 6.96 & 1444666.45 & 8940.45 & 5450.91 & 234.89 & 2019 \\
\hline 4.14 & 893209.27 & 4964.45 & 2434.89 & 91.52 & المتوسط \\
\hline
\end{tabular}




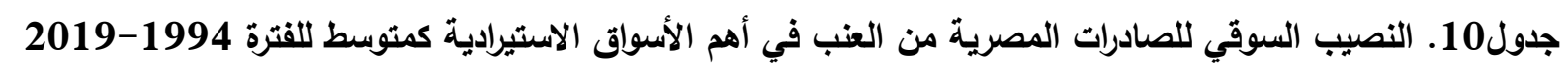

\begin{tabular}{|c|c|c|c|}
\hline السوقيّب\% & إجمالي وارداتثف الدولةّة من العنب & كميةٌ الصادرات المصريةٌ من العنب & الدولة - الة \\
\hline 12.86 & 215.66 & 27.74 & بريطانيا وشمال ايرلندا \\
\hline 6.28 & 252.57 & 15.86 & هولندا \\
\hline 2.46 & 326.49 & 8.04 & إلمانيا \\
\hline 43.10 & 18.31 & 7.89 & أيطاليا \\
\hline 3.16 & 231.90 & 7.32 & روسياً الاتحادية \\
\hline 6.57 & 70.27 & 4.62 & بلجيكا \\
\hline 9.84 & 34.90 & 3.43 & الإمارات ألعربية المتحدة \\
\hline 27.98 & 8.98 & 2.51 & سلوفينيا \\
\hline 6.64 & 37.10 & 2.46 & السعودية \\
\hline 61.16 & 3.23 & 1.97 & السودان \\
\hline
\end{tabular}

WWw.fao.org المصدر : جمعت وحسبت من - موقع منظمة الأغذية والزراعة

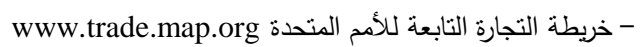

وهولندا وروسيا والمانيا النصيب السوقي للعنب المصري فيهم منخفض مما يتطلب دراسة إمكانيات زيادة النصيب السوقي وهي

$$
\text { بتلك الدول. }
$$

\section{المراجع}

المنظمة العربية للتمية الزراعية، دليل الممارسات الزراعية الجيدة

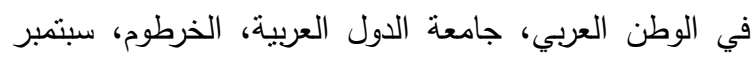
2007

خريطة التجارة التابعة للأمم المتحدة www.trade.map.org. عبد الرزاق بلعباس، من المعايير الضائعة في الفكر المقاصدي: التتبع، مجلة التجديد، المجلد (22)، العدد (43)، الجامعة لرئة

$$
\text { الإسلامية العالمية ماليزيا، } 2018 .
$$

عصام محمد يوسف عبد العال، دراسة اقتصادية لنظام التتبع ودوره في تتمية الصادرات الزراعية في مصر ، رسالة دكنوراه، قسم

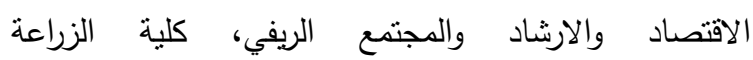

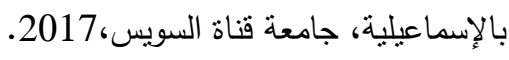

قانون الهيئة القومية لسلامة الغذاء، قانون رقم 1 لسنة 2017، قرار رئيس مجلس الوزراء رقم 412 لسنة 2019، الجريدة الرسمية، العدد 1، يناير 2017.
مؤثر النصيب السوقي للصادرات المصرية من العنب في

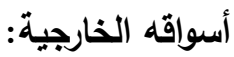
تبين من جدول (10) أن السوق السوداني والسوق الإيطالي يتصدروا قائمة الأسواق المستوردة للعنب المصري، حيث بلغ النصيب السوقي لكل منها حوالي 61.16\%؛ 43.10\% على الترتيب من متوسط إجمالي واردات تلاتك الدول من العنب. كما تحظى أسواق كل من سلوفينيا وبريطانيا وشمال ايرلندا والإمارات العربية المتحدة والسعودية

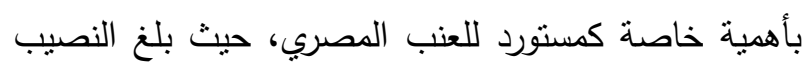
السوقي لكل منها حوالي 27.98\%، 12.86\%، 9.84؛ 6.64 على الترتيب من متوسط إجمالي واردات نلك الدول من العنب، يليها كلا من بلجيكا وهولندا حيث بلغ النصيب السوقي لكل منها حوالي 6.57\%، 6.28\% على الترنيب من متوسط إجمالي واردات تللك الدول من العنب. كما تبين تزايد كمية وادات كلا من روسيا والمانيا من العنب وعلى الرغم من ذلك مازال نصيب مصر السوقي

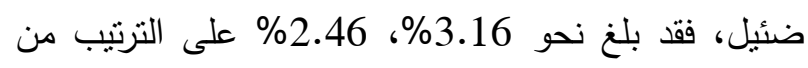
متوسط واردات السوق الروسي والسوق الالماني خلال منوسط الفترة 1994- 2019. ومن ذلك تبين أن بلجيكا 
Rini Banerjee, ITC Intern, and Hema Menon, An Overview of Traceability in Food and Agricultural Products, International Trade Centre (ITC), Geneva, Switzerland ,2015.

$$
\begin{aligned}
& \text { مشيرة محمد عبد المجيد البطران، دراسة قياسية للعوامل المؤثرة } \\
& \text { على نظام التتبع مع الاتحاد الأوروبي (دراسة حالة لمحصولي دراسل }
\end{aligned}
$$

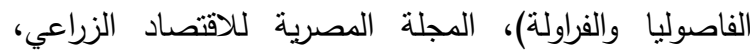

$$
\begin{aligned}
& \text { المجلد (29)، العدد (1)، مارس } 9 \text { ا.ب. } \\
& \text { موقع منظمة الأغذية والزراعة العالمية (الفاو) www.fao.org. }
\end{aligned}
$$

\title{
ABSTRACT \\ The Impact of the Application of the Traceability System on Egypt's Exports of Grapes
}

\author{
Ahmed Abou El-Yazid El-Rasoul, Sameh Mohamed Hassan Shehab, Mai Mostafa Hassan Morsi and \\ Fatma Elzhraa Sabry Mohamed Aly
}

The research aimed to identify the impact of the application of the traceability system on increasing the competitiveness and growth of Egyptian exports of grapes to the European Union countries by identifying the current status of grape exports to the European Union through the geographical distribution of Egyptian exports, as well as the most important indicators of measuring the competitiveness of Egypt's exports of grapes. To determine the impact of the application of the traceability system on stimulating Egyptian exports of grapes during the period 1994-2019. This period was divided into two periods, the first period 1994-2006, which is the period before the application of the traceability system, and the second period 2007-2019, which is the period after the implementation of the traceability system for crops. agricultural export in Egypt.

Key words: Traceability system, Egyptian export, graps, European Union, competitiveness, indicators. 